\title{
Modes Orthogonality of the Mechanical System Simple Supported Beam-Actuators-Concentrated Masses
}

\author{
A. BRAŃSKI* \\ Laboratory of Acoustics, Department of Electrical and Computer Engineering, Rzeszów University of Technology \\ Wincentego Pola 2, 35-959 Rzeszów, Poland
}

\begin{abstract}
This paper deals with simple supported beam-actuators-concentrated masses mechanical system; it appears in active vibration reduction problem. To solve the problem with the Fourier method, the system is discretized into uniform elements. In the paper the orthogonality condition of the modes of the discretized system is derived. Furthermore, the solution of the forced vibration problem of the above system, appearing inherently in the active vibration reduction problem, is outlined.
\end{abstract}

PACS: 46.70.De, 77.84.-s, 43.20.Tb

\section{Introduction}

The beam, actuators and (concentrated) masses can consist of three mechanical systems as the research objects. First of them makes up beam-masses, the second: beam-actuators and the last is a combination above mentioned ones, i.e. beam-actuators-masses (BAM).

The beam is a structural element carrying masses elastically or stiffly attached to it; this is the natural task of the beam. The concentrated masses are also used to the modification of the structure dynamics $[1,2]$. The free vibration of these systems are solved in many papers, but only natural frequencies or/and modes have usually been obtained by means of an exact analysis [2, 3], as well as by employing some approximate methods $[4,5]$.

Second mechanical system, the beam-actuators, is of practical interest in active vibration reduction [6-11]. Actuators are glued to the beam and they become part of it. Both actuators and the glue (layers) are relatively light, compared to the structure. However, to make the vibrating simulation more precise, the dynamic effects (mass loading and stiffness) of actuators and glue should be considered. This problem is solved numerically: for actuators in $[12,13]$, for glue in $[14,15]$.

The BAM system appears in active beam vibration reduction with masses. Adding actuators (and the glue at the same time) is the technical necessity. As far as masses are concerned, from practical point of view, the beam is designed for loading with the masses.

Furthermore, from a scientific point of view, adding them may be substantiated as follows. To clearly explain the optimal distribution of the actuators on the beam in active vibration reduction, the asymmetrical forms are

* e-mail: abranski@prz.rzeszow.pl needed. The asymmetrical effect may be achieved adding to the beam concentrated masses. This problem is widely explained in [16] and presented in [17].

Now the problem arises, how to describe the free and forced vibrations of the above systems? It may be solved as follows. The effects of attached masses are expressed with the Dirac delta distribution, but dynamic effects of bonded actuators and glue are expressed with the Heaviside one. So, the theory of distribution is used to formulate this problem [18]. Next, the differential equation of transverse vibrations of the BAM system with a local, stroke change of stiffness is derived.

Another attitude, admittedly applied to the uniform beam, is presented in [19]. The uniform beam is discretized into elements. The boundary conditions of each individual element are modeled by using translational and rotational spring and concentrated mass. The study in [19] is restricted to the natural frequencies and the mode shapes of the beam. This paper is an extension of the above reference. Here, the system BAM is not uniform, but it is discretized into uniform elements. The division is not coincidental; it is imposed out of the change of physical properties, i.e. properties of actuators, glue and masses. To solve the free and forced vibrations problem of the BAM discretized system with the Fourier method (separation of variables method, solution by eigenfunction expansion), the eigenfrequencies (natural frequencies), eigenfunctions (modes) and their orthogonality condition are needed. The aim of the paper is just to derive the orthogonality condition of the modes.

\section{Modal analysis formulation}

Let be the simple supported beam as depicted in Fig. 1 . The particular form of the Bernoulli-Euler equation gov- 
erning transverse modal vibration has a following standard form ([20] s. 172, [21]),

$$
E J \mathrm{D}^{4} u+\rho S \mathrm{D}_{t}^{2} u=0,
$$

where $u=u(x, t)$ - beam deflection at the point $x$ and the time $t, \mathrm{D}^{4}()=.\partial^{4}(.) / \partial x^{4}, \mathrm{D}_{t}()=.\partial(.) / \partial t, b$ - beam width; hereafter the rest symbols are explained together.

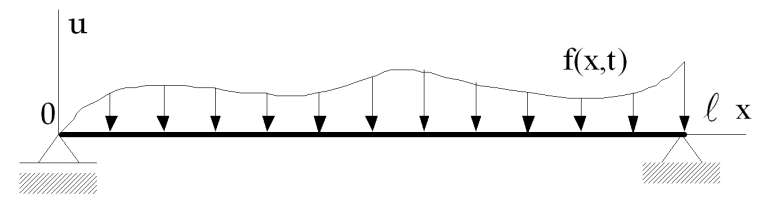

Fig. 1. The geometry of the simple supported beam.

To solve Eq. (2.1) explicitly, four boundary conditions at the ends of the beam are needed. For the simple supported beam both displacement and the bending moment are equal to zero

$$
\begin{aligned}
& u(0, t)=0, \quad \mathrm{D}^{2} u(0, t)=0, \\
& u(\ell, t)=0, \quad \mathrm{D}^{2} u(\ell, t)=0 .
\end{aligned}
$$

Furthermore, one needs to know the initial conditions. They represent the initial displacement and the speed of the beam points

$$
\begin{aligned}
& u(x, 0)=\hat{u}(x), \\
& \mathrm{D}_{t} u(x, 0)=\hat{v}(x) .
\end{aligned}
$$

Equations from (2.1) to (2.5) constitute the modal analysis formulation.

\subsection{Beam with actuators}

To solve the modal problem of the BAM system, Eq. (2.1) must be rounded out. First of all, actuators must be glued to the beam; they are arranged as depicted in Fig. 2. The location and length of separate actuators (and the glue layers simultaneously), are denoted commonly with coordinates $\left\{x_{s}\right\}$ and $\left\{\ell_{s}\right\}$ respectively, $s=1,2, \ldots, n_{s}$.

Fig. 2. Distribution of actuators and glue layers on the beam.

Let $P=\{E, J, h, \rho, S\}$ means the physical and geometrical parameters of the beam, actuators and glue, i.e. \{Young's modulus, moment of inertia of the cross-section, thickness, mass density, area of the cross-section\}, respectively. To be more precise, all parameters are supplemented with additional index $\vartheta=$ $\{b, a, g\}=\{[b]$ eam, $[a]$ ctuator, $[g]$ lue $\}$, for example, $J_{\vartheta}$ means moments of inertia of the surface cross-sections. The parameters of the BAM system may be written as

$$
P=P_{b}+\sum_{s} P_{s} H\left(x_{1 s}-x_{2 s}\right)=P_{b}+\sum_{s} P_{s}\langle H\rangle^{0},
$$

where $P_{s}=P_{a}+P_{g},\langle H\rangle^{0}=H\left(x_{1 s}-x_{2 s}\right)=H\left(x-x_{1 s}\right)-$ $H\left(x-x_{2 s}\right), H\left(x-x_{1 s}\right)$ - the Heaviside step function in point $x_{1 s}$ and so on, $\left\{x_{1 s}, x_{2 s}\right\}=\left\{x_{s}-\ell_{s} / 2, x_{s}+\ell_{s} / 2\right\}$.

Considering dynamic effects of the actuators and glue on the beam vibration, Eq. (2.1) takes the form

$$
\begin{aligned}
& \left(E_{b} J_{b}+\sum_{s} E_{s} J_{s}\langle H\rangle^{0}\right) \mathrm{D}^{4} u \\
& \quad+\left(\rho_{b} S_{b}+\sum_{s} \rho_{s} S_{s}\langle H\rangle^{0}\right) D_{t}^{2} u=0 .
\end{aligned}
$$

Equation (2.7) may be written down exactly the same like Eq. (2.1), if one denotes

$$
\begin{aligned}
& E J=E_{b} J_{b}+\sum_{s} E_{s} J_{s}\langle H\rangle^{0}, \\
& \rho S=\rho_{b} S_{b}+\sum_{s} \rho_{s} S_{s}\langle H\rangle^{0},
\end{aligned}
$$

where $E J$ - equivalent bending stiffness, $J_{s}$ - cross-sectional moment of inertia of the $s$-th layer relative to the mid-plane [15].

\subsection{Beam with masses}

This problem corresponds to the one given in previous subsection; it is not the same, however, there are many similarities. Let a few masses be attached to the beam $[22,1]$. They are marked by $\left\{m_{r}\right\}, r=1,2, \ldots, n_{r}$ and their distribution is described with set of coordinates $\left\{x_{r}\right\}$, see Fig. 3, hence

$$
\begin{aligned}
& \alpha_{r}=\sum_{r} m_{r} \delta\left(x-x_{r}\right)=m_{1} \delta\left(x-x_{1}\right) \\
& \quad+m_{2} \delta\left(x-x_{2}\right)+\ldots+m_{r} \delta\left(x-x_{r}\right)+\ldots
\end{aligned}
$$

where $\delta($.$) - the Dirac delta distribution.$

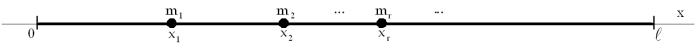

Fig. 3. Distribution of the concentrated masses on the beam.

The dynamic effects of the $\left\{m_{r}\right\}$ may be include in Eq. (2.1), hence

$$
E J \mathrm{D}^{4} u+\left(\rho S+\alpha_{r}\right) \mathrm{D}_{t}^{2} u=0 .
$$

\subsection{Beam with actuators and masses}

If the $E J, \rho S$ take the form given by Eq. (2.8), Eq. (2.10) governs the modal vibration of the BAM system and in explicit form one has

$$
\begin{aligned}
& \left(E_{b} J_{b}+\sum_{s} E_{s} J_{s}\langle H\rangle^{0}\right) \mathrm{D}^{4} u \\
& +\left(\rho_{b} S_{b}+\sum_{s} \rho_{s} S_{s}\langle H\rangle^{0}+\alpha_{r}\right) \mathrm{D}_{t}^{2} u=0 .
\end{aligned}
$$

On the ground of the $E J, \rho S$ and $\alpha_{r}$ form, Eq. (2.11) cannot be understood in a classical manner. To solve it, 
some methods may be applied. One of them is presented in [1, 4, 18]; another attitude may be found in [19] and it is applied here.

\section{Solution of the modal problem}

At the attitude to the modal analysis given in [19], the system is divided into $j$ elements, $j=1,2, \ldots, n_{j}$, Fig. 4 . No losses of the generality, masses are attached to the beam at the ends of actuators. So, the discretization is imposed by masses. Hence, the elements between masses become uniform, i.e. both $E J$ and $\rho S$, described with Eq. (2.8), are constant. All elements may be considered separately in local coordinates, $x \in\left[0, e_{j}\right)$, Fig. 5 , and the solution to Eq. (2.11) can be expressed as

$$
u(x, t)=\sum_{j} u_{j}(x, t),
$$

where $u_{j}(x, t)$ fulfills the equation, which arise out of Eq. (2.11),

$$
E_{j} J_{j} \mathrm{D}^{4} u_{j}(x, t)+\rho_{j} S_{j} \mathrm{D}_{t}^{2} u_{j}(x, t)=0,
$$

where $E_{j} J_{j}$ and $\rho_{j} S_{j}$ are different on the separate elements and they are given by Eq. (2.8).

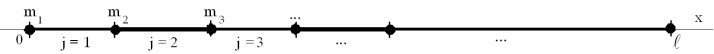

Fig. 4. Geometry of the general BAM system.

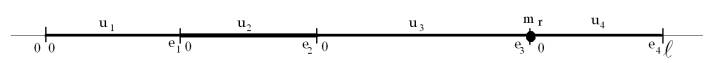

Fig. 5. Geometry of the general BAM system in local coordinates.

As can be seen from Eq. (3.2), the dynamic effect of the actuators and glue layers only are taken into account, but this effect of the masses is omitted. This is because the concentrated masses $\left\{m_{r}\right\}$ are considered in boundary conditions for the $j$ elements.

The boundary conditions for the $j$ elements consist of boundary conditions of the problem and coupling ones. The latter are obtained by considering continuity of displacement, slope, the relationship between the shear force and bending moment at the points between neighboring elements.

Let the solution to Eq. (3.2) be represented by a product of spatial and temporal functions

$$
u_{j}(x, t)=X_{j}(x) T(t) .
$$

Substitute Eq. (3.3) into Eq. (3.2), and bring terms containing $x$ to the left hand side (LHS) and terms containing $t$ to the right hand side (RHS), then one obtains

$$
\frac{E_{j} J_{j}}{\rho_{j} S_{j}} \frac{\mathrm{D}^{4} X_{j}(x)}{X_{j}(x)}=-\frac{\mathrm{D}_{t}^{2} T(t)}{T(t)}=\omega^{2},
$$

where $\omega$ is defined as a constant and it is a natural frequency of the BAM system.
Equation (3.4) corresponds to the two ordinary differential equations, namely

$$
\begin{aligned}
& \mathrm{D}^{4} X_{j}(x)-\lambda_{j}^{4} X_{j}(x)=0, \\
& \mathrm{D}_{t}^{2} T(t)+\omega^{2} T(t)=0,
\end{aligned}
$$

where the dispersion relationship to Eq. (3.5) is given by

$$
\lambda_{j}^{4}=\omega^{2} \frac{\rho_{j} S_{j}}{E_{j} J_{j}}=\frac{\omega^{2}}{\gamma_{j}} .
$$

The solution of above equations provides the natural frequencies and the corresponding modes. The $\lambda_{j}$ are different on separate elements and they are yet to be determined. Furthermore, the $\lambda_{j}$ depend on $\omega$ which is unchanging for all BAM system.

In view of mnemonic way of differentiation and integration of the Krylov functions, [20], the general solution to Eq. (3.5) is given by

$$
\begin{aligned}
& X_{j}(x)=A_{j} K_{1}\left(\lambda_{j} x\right)+B_{j} K_{2}\left(\lambda_{j} x\right)+C_{j} K_{3}\left(\lambda_{j} x\right) \\
& \quad+D_{j} K_{4}\left(\lambda_{j} x\right)
\end{aligned}
$$

where

$$
\begin{aligned}
& K_{1}(z)=[\operatorname{ch}(z)+\cos (z)] / 2, \\
& K_{2}(z)=[\operatorname{sh}(z)-\sin (z)] / 2, \\
& K_{3}(z)=[\operatorname{ch}(z)-\cos (z)] / 2, \\
& K_{4}(z)=[\operatorname{sh}(z)+\sin (z)] / 2 .
\end{aligned}
$$

The boundary conditions to the separate $j$ element in local coordinates $x \in\left[0, e_{j}\right]$ and harmonic steady state, have the general form:

- boundary conditions at the left end of the 1 st element

$X_{1}(0)=0$,

$\mathrm{D}^{2} X_{1}(0)=0$.

- coupling conditions between $j$-th and $(j+1)$-th elements, Fig. 6,

$$
\begin{aligned}
& X_{j}\left(\lambda_{j} e_{j}\right)=X_{j+1}(0), \\
& \mathrm{D} X_{j}\left(\lambda_{j} e_{j}\right)=\mathrm{D} X_{j+1}(0), \\
& E_{j} J_{j} \mathrm{D}^{2} X_{j}\left(\lambda_{j} e_{j}\right)=E_{j+1} J_{j+1} \mathrm{D}^{2} X_{j+1}(0), \\
& E_{j} J_{j} \mathrm{D}^{3} X_{j}\left(\lambda_{j} e_{j}\right)+m_{j+1} \omega^{2} X_{j}\left(\lambda_{j} e_{j}\right) \\
& \quad=E_{j+1} J_{j+1} \mathrm{D}^{3} X_{j+1}(0),
\end{aligned}
$$

or

$$
\begin{aligned}
& E_{j} J_{j} \mathrm{D}^{3} X_{j}\left(\lambda_{j} e_{j}\right) \\
& \quad=m_{j+1} \omega^{2} X_{j+1}(0)+E_{j+1} J_{j+1} \mathrm{D}^{3} X_{j+1}(0) .
\end{aligned}
$$

From numerical point of view, the last identity in Eq. (3.11) is more convenient.

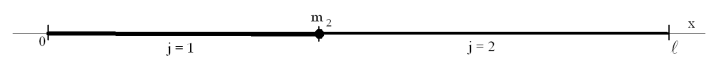

Fig. 6. Coupling conditions between 1st and 2nd elements.

- boundary conditions at the right end of the $n_{j}$-th element

$$
X_{n_{j}}\left(e_{n_{j}}\right)=0 \text {, }
$$




$$
\mathrm{D}^{2} X_{n_{j}}\left(e_{n_{j}}\right)=0 \text {. }
$$

Since $\lambda_{1} \neq \lambda_{2} \neq \ldots \neq \lambda_{j} \neq \ldots$ then, to calculate them, Eq. (3.7) must be used. It is convenient to express $\left\{\lambda_{2}, \lambda_{3}, \ldots, \lambda_{j}, \ldots\right\}$ as a function $\lambda_{1}$, hence

$$
\lambda_{1}^{4} \gamma_{1}=\lambda_{2}^{4} \gamma_{2}=\ldots=\lambda_{j}^{4} \gamma_{j}=\ldots=\omega^{2}
$$

or

$$
\begin{aligned}
& \lambda_{2}^{4}=\lambda_{1}^{4}\left(\gamma_{1} / \gamma_{2}\right), \\
& \lambda_{3}^{4}=\lambda_{1}^{4}\left(\gamma_{1} / \gamma_{3}\right), \ldots \lambda_{j}^{4}=\lambda_{1}^{4}\left(\gamma_{1} / \gamma_{j}\right), \ldots .
\end{aligned}
$$

Substituting Eq. (3.8) into boundary conditions (3.10) it appears that $A_{1}=0, C_{1}=0$. In the same way, the rest of conditions given by Eqs. (3.11), (3.12) leads to the set of algebraic equations and it may be written in the matrix form

$$
\boldsymbol{A a}=\mathbf{0} .
$$

In general, the matrix $\boldsymbol{A}$ is too large to present it in explicit form. A simple example is considered in Sect. 5.

\section{Orthogonality condition}

This property of the uniform beam modes may be found in [20, 21]. First of all, the identity is derived, i.e. based on twice integration by parts, one has

$$
\begin{aligned}
& \int_{0}^{\ell} X_{\nu} \mathrm{D}^{4} X_{\mu} \mathrm{d} x=\left.\left(X_{\nu} \mathrm{D}^{3} X_{\mu}-\mathrm{D} X_{\nu} \mathrm{D}^{2} X_{\mu}\right)\right|_{0} ^{\ell} \\
& \quad+\int_{0}^{\ell} \mathrm{D}^{2} X_{\nu} \mathrm{D}^{2} X_{\mu} \mathrm{d} x .
\end{aligned}
$$

For simplicity, here and in the future, an argument $(x)$ is omitted. The separate modes $X_{\mu}(x)$ and $X_{\nu}(x)$ fulfill the modal equations, cf. Eq. (3.5). For convenience they are written down in expanded form

$$
\begin{aligned}
& E_{J D^{4} X_{\nu}(x)}=\omega_{\nu}^{2} \rho S X_{\nu}(x), \\
& E^{4} X_{\mu}(x)=\omega_{\mu}^{2} \rho S X_{\mu}(x) .
\end{aligned}
$$

Multiplying above equations by $X_{\nu}(x)$ and $X_{\mu}(x)$, respectively, integrate both in range of integration $x \in$ $[0, \ell]$, use Eq. (4.1), subtract the second result from the first one and then one obtains

$$
\begin{aligned}
& \left(\omega_{\nu}^{2}-\omega_{\mu}^{2}\right) \rho S \int_{0}^{\ell} X_{\nu} X_{\mu} \mathrm{d} x \\
& \quad=E J\left[\left(X_{\mu} \mathrm{D}^{3} X_{\nu}-\mathrm{D} X_{\mu} \mathrm{D}^{2} X_{\nu}\right)\right. \\
& \left.-\left(X_{\nu} \mathrm{D}^{3} X_{\mu}-\mathrm{D} X_{\nu} \mathrm{D}^{2} X_{\mu}\right)\right]\left.\right|_{0} ^{\ell} .
\end{aligned}
$$

For standard boundary conditions, the right-hand-side equals zero, and standard form of the orthogonality condition is obtained.

The above orthogonality condition may be adapted to the BAM system in a simple way. Let the system be divided into $n_{j}$ elements as depicted in Fig. 4. Now, Eq. (4.1) must be applied to the separate $j$ element, namely

$$
\begin{aligned}
& \int_{0}^{e_{j}} X_{j \nu} \mathrm{D}^{4} X_{j \mu} \mathrm{d} x=\left.\left(X_{j \nu} \mathrm{D}^{3} X_{j \mu}-\mathrm{D} X_{j \nu} \mathrm{D}^{2} X_{j \mu}\right)\right|_{0} ^{e_{j}} \\
& \quad+\int_{0}^{e_{j}} \mathrm{D}^{2} X_{j \nu} \mathrm{D}^{2} X_{j \mu} \mathrm{d} x .
\end{aligned}
$$

and coupling conditions between neighboring elements, Eqs. (3.10)-(3.12), instead of Eq. (4.4) one has

$$
\begin{aligned}
& \left(\omega_{\nu}^{2}-\omega_{\mu}^{2}\right)\left(\rho_{1} S_{1} \int_{0}^{e_{1}} X_{1 \nu} X_{1 \mu} \mathrm{d} x+\ldots+\rho_{n_{j}} S_{n_{j}}\right. \\
& \quad \times \int_{0}^{e_{n_{j}}} X_{n_{j} \nu} X_{n_{j} \mu} \mathrm{d} x+m_{1} X_{1 \nu}(0) X_{1 \mu}(0)+\ldots \\
& \quad+m_{n_{j}} X_{n_{j} \nu}(0) X_{n_{j} \mu}(0) \\
& \left.+m_{n_{j}+1} X_{n_{j} \nu}\left(e_{n_{j}}\right) X_{n_{j} \mu}\left(e_{n_{j}}\right)\right) \\
& =E_{n_{j}} J_{n_{j}}\left[\left(X_{n_{j} \mu}\left(e_{n_{j}}\right) \mathrm{D}^{3} X_{n_{j} \nu}\left(e_{n_{j}}\right)\right.\right. \\
& \left.-\mathrm{D} X_{n_{j} \mu}\left(e_{n_{j}}\right) \mathrm{D}^{2} X_{n_{j} \nu}\left(e_{n_{j}}\right)\right) \\
& -\left(X_{n_{j} \nu}\left(e_{n_{j}}\right) \mathrm{D}^{3} X_{n_{j} \mu}\left(e_{n_{j}}\right)\right. \\
& \left.\left.-\mathrm{D} X_{n_{j} \nu}\left(e_{n_{j}}\right) \mathrm{D}^{2} X_{n_{j} \mu}\left(e_{n_{j}}\right)\right)\right] .
\end{aligned}
$$

Because of Eq. (3.12), the right-hand-side is zero. Furthermore, the $m_{1}$ and $m_{n_{j}+1}$ contribute no dynamic effects and they may be omitted. So, the general orthogonality condition is given by

$$
\begin{gathered}
\left(\omega_{\nu}^{2}-\omega_{\mu}^{2}\right) \sum_{j}\left(\rho_{j} S_{j} \int_{j} X_{j \nu}(x) X_{j \mu}(x) \mathrm{d} x\right. \\
\left.+m_{j+1} X_{j+1 \nu}(0) X_{j+1 \mu}(0)\right)=0 .
\end{gathered}
$$

Since the term $\omega_{\nu}^{2}-\omega_{\mu}^{2}$ is cancelled for $\mu=\nu$, one has

$$
\begin{aligned}
& \sum_{j}\left(\rho_{j} S_{j} \int_{j} X_{j \nu}(x) X_{j \mu}(x) \mathrm{d} x\right. \\
& \left.\quad+m_{j+1} X_{j+1 \nu}(0) X_{j+1 \mu}(0)\right)=\left\{\begin{array}{l}
0, \nu \neq \mu, \\
\beta_{\nu}, \nu=\mu .
\end{array}\right.
\end{aligned}
$$

Since the $m_{j}$ at $x=\{0, \ell\}$ may be omitted a priori, the masses may be described as depicted in Fig. 7. For this case, instead of Eq. (4.8), the orthogonality condition for the BAM system is given by

$$
\begin{aligned}
& \sum_{j}\left(\rho_{j} S_{j} \int_{j} X_{j \nu}(x) X_{j \mu}(x) \mathrm{d} x+m_{j} X_{j \nu}\left(e_{j}\right) X_{j \mu}\left(e_{j}\right)\right) \\
& =\left\{\begin{array}{l}
0, \nu \neq \mu, \\
\beta_{\nu}, \nu=\mu,
\end{array}\right.
\end{aligned}
$$

where $m_{n_{j}}$ ought to be assumed equal to zero.

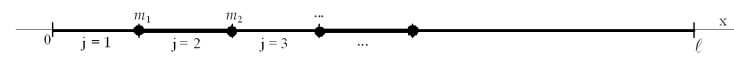

Fig. 7. Geometry of $n_{j}$ elements and masses which take an active part in the dynamic effect.

Both Eqs. (4.8) and (4.9) assure the same results, but the former is numerically faster. Generally speaking, either Eq. (4.8) or Eq. (4.9) may be used in deriving the solution to the forced vibration problem.

Considering both boundary conditions of the problem 


\section{Examples}

\subsection{A simple modal problem}

Let consider the problem presented in Fig. 6, where $j=1,2,\left\{m_{r}\right\}=\left\{m_{1}, m_{2}, m_{3}\right\}$. Since $m_{1}$ and $m_{3}$ are placed at the ends of the simple supported beam, their dynamic effects are equal to zeros. So, only $m_{2}$ should be considered. In this simple case one has

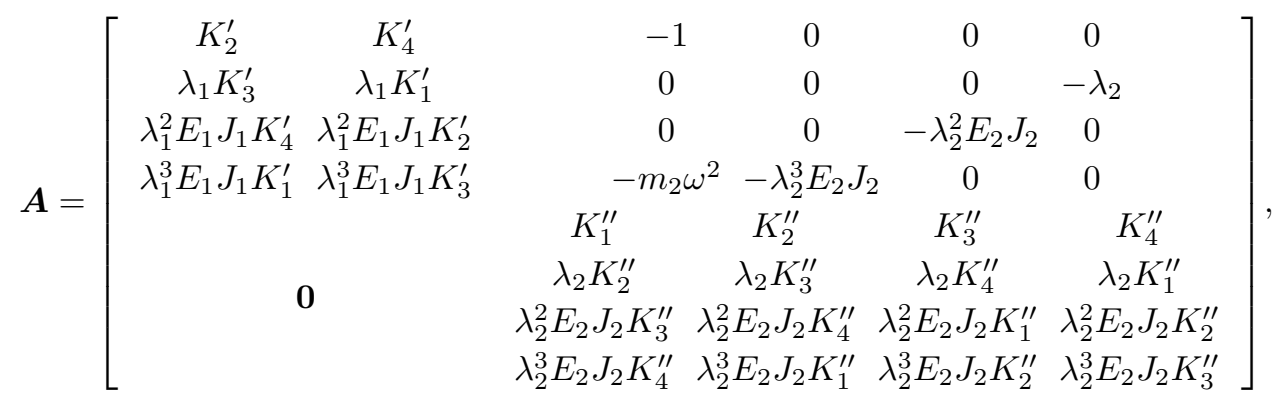

where the symbols in matrices are given by

$$
\begin{aligned}
& \left\{K_{v}\right\}=\left\{K_{1}, K_{2}, K_{3}, K_{4}\right\}, \quad K_{v}^{\prime}=K_{v}\left(\lambda_{1} e_{1}\right), \\
& K_{v}^{\prime \prime}=K_{v}\left(\lambda_{2} e_{2}\right) .
\end{aligned}
$$

The unknowns are collected in column matrix

$$
\boldsymbol{a}=\left[B_{1}, D_{1}, A_{2}, B_{2}, C_{2}, D_{2}\right]^{\mathrm{T}} .
$$
It seems that this simple example well conveys the creating idea of the main matrix $\boldsymbol{A}$.

To solve Eq. (3.15), one assumes that $\operatorname{det} \boldsymbol{A}\left(\lambda_{1}\right)=0$ and it leads to the frequency equation. As the result, the set $\left\{\lambda_{1 \nu}\right\}, \nu=1,2, \ldots, n$ is obtained. Based on Eq. (3.14), one can calculate $\left\{\lambda_{2 \nu}, \lambda_{3 \nu}, \ldots, \lambda_{j \nu}, \ldots\right\}$ and finally, based on Eq. (3.7), the natural frequencies $\left\{\omega_{\nu}\right\}$ of the BAM system.

Now, the unknowns $\boldsymbol{a}$, Eq. (5.3), should be determined. Let the main matrix elements be written as two suffix quantities $A_{\alpha \beta}$, where $\alpha$ and $\beta$ label the rows and columns respectively. Let $M_{\alpha \beta}$ be the minor of the $A_{\alpha \beta}$ element. The general solution to Eq. (3.15) is

$$
\begin{aligned}
& B_{1}: D_{1}: A_{2}: \ldots \\
& \quad=(-1)^{\alpha+1} M_{\alpha 1}:(-1)^{\alpha+2} M_{\alpha 2}:(-1)^{\alpha+3} M_{\alpha 3}: \ldots
\end{aligned}
$$

Substituting $\left\{\lambda_{j \nu}\right\}$ and unknowns $\boldsymbol{a}$ to Eq. (3.8), the $\nu$ modes assigned to the $j$ element are obtained. The solution to Eq. (3.5) for the BAM system is given by

$$
\begin{aligned}
& X(x)=\sum_{j} X_{j}(x)=\sum_{j \nu} X_{j}\left(\lambda_{j \nu} x\right)=\sum_{j \nu} X_{j \nu}(x) \\
& \quad=\sum_{\nu} X_{\nu}(x)
\end{aligned}
$$

where $\sum_{j \nu}(\ldots)=\sum_{j} \sum_{\nu}(\ldots)$ and the separate modes on $j$ element are equal to

$$
\begin{aligned}
& X_{j \nu}(x)=A_{j} K_{1}\left(\lambda_{j \nu} x\right)+B_{j} K_{2}\left(\lambda_{j \nu} x\right)+C_{j} K_{3}\left(\lambda_{j \nu} x\right) \\
& \quad+D_{j} K_{4}\left(\lambda_{j \nu} x\right) .
\end{aligned}
$$

The $\nu$ modes $X_{\nu}(x)$ are taken for future considerations.

\subsection{Forced vibrations of the beam}

To analyze the forced vibrations, the orthogonality condition would have to be used. This analysis is realized in some steps. In the following they are presented, omitting everywhere for simplicity, the subscript $j$. So, the analysis is much the same like for the uniform beam.

1. Let be the (nonhomogeneous) equation of the forced beam vibration

$$
\begin{aligned}
& E J\left(\mathrm{D}^{4} u_{f}(x, t)+\mu_{d} \mathrm{D}^{4}\left(\mathrm{D}_{t} u_{f}(x, t)\right)\right)+\rho S \mathrm{D}_{t}^{2} u_{f}(x, t) \\
& \quad=-f(x, t),
\end{aligned}
$$

where hereafter $u_{f}(x, t)=u_{f ; j}(x, t)$ - forced vibration of the $j$ element, $\mu_{d}$ - inner damping factor.

2. To find the forced solution, apply the Fourier method

$$
u_{f}(x, t)=\sum_{\nu} u_{f ; \nu}(x, t)=\sum_{\nu} X_{\nu}(x) T_{\nu}(t) .
$$

3. Substitute Eq. (5.8) in Eq. (5.7), hence

$$
\begin{aligned}
& E J \sum_{\nu} \mathrm{D}^{4} X_{\nu}(x)\left(\mu_{d} \mathrm{D}_{t} T_{\nu}(t)+T_{\nu}(t)\right) \\
& \quad+\rho S \sum_{\nu} X_{\nu}(x) \mathrm{D}_{t}^{2} T_{\nu}(t)=-f_{x}(x) f_{t}(t),
\end{aligned}
$$

where the excited force is assumed as $f(x, t)=f_{x}(x) f_{t}(t)$.

4. Apply to the first term on the LHS Eq. (3.5): here $\mathrm{D}^{4} X_{\nu}(x)=\lambda_{\nu}^{4} X_{\nu}(x)$, divide by $\rho S$, apply the dispersion condition, Eq. (3.7): here $\lambda_{\nu}^{4}=(\rho S / E J) \omega_{\nu}^{2}$; it leads to

$$
\begin{aligned}
& \sum_{\nu} \omega_{\nu}^{2} X_{\nu}(x)\left[\mu_{d} \mathrm{D}_{t} T_{\nu}(t)+T_{\nu}(t)\right]+\sum_{\nu} X_{\nu}(x) \mathrm{D}_{t}^{2} T_{\nu}(t) \\
& \quad=-\frac{1}{\rho S} f_{x}(x) f_{t}(t) .
\end{aligned}
$$

5. Multiple by $X_{\mu}(x)$ and integrate with respect to $x \in[0, \ell]$. At just this step, it is the first time the orthogonality condition Eq. (4.4) is used. It leads to the time equation

$$
\mathrm{D}_{t}^{2} T_{\nu}(t)+\mu_{d} \omega_{\nu}^{2} \mathrm{D}_{t} T_{\nu}(t)+\omega_{\nu}^{2} T_{\nu}(t)=\overline{\mathrm{D}}_{\nu} f_{t}(t),
$$

where 


$$
\overline{\mathrm{D}}_{\nu}=\frac{1}{\rho S} \frac{1}{\beta_{\nu}} I_{\nu}, \quad I_{\nu}=-\int_{0}^{\ell} f_{x}(x) X_{\nu}(x) \mathrm{d} x .
$$

6. Define the initial condition for $T_{\nu}(t)$, for example

$$
\begin{aligned}
& u(x, 0)=\left.u(x, t)\right|_{t=0}=\hat{u}(x), \\
& D_{t} u(x, 0)=\left.D_{t} u(x, t)\right|_{t=0}=\hat{v}(x) .
\end{aligned}
$$

7. Substitute Eq. (5.8) in Eq. (5.13), multiple by $X_{\mu}(x)$ and integrate with respect to $x \in[0, \ell]$. At this step, it is the second time the orthogonality condition Eq. (4.4) is used; it leads to

$$
\begin{aligned}
& T_{\nu}(0)=\frac{1}{\beta_{\nu}} \int_{0}^{\ell} \hat{u}(x) X_{\nu}(x) \mathrm{d} x, \\
& D_{t} T_{\nu}(0)=\frac{1}{\beta_{\nu}} \int_{0}^{\ell} \hat{v}(x) X_{\nu}(x) \mathrm{d} x .
\end{aligned}
$$

8. Assume the RHS of Eq. (5.11) in the form

$$
f_{t}(t)=\exp \left(\mathrm{i} \omega_{f} t\right),
$$

where $\omega_{f}-$ forced annular frequency, $\mathrm{i}=(-1)^{1 / 2}$.

9. For the sake of Eq. (5.15), the suitable solution of nonhomogeneous time Eq. (5.11) is

$$
T_{\nu}(t)=\bar{C} \exp \left(\mathrm{i} \omega_{f} t\right),
$$

where $\bar{C}$ - unknown constant

10. Substitute Eqs. (5.15) and (5.16) in Eq. (5.11), hence

$$
\bar{C}=\bar{C}_{\nu}=\frac{1}{\omega_{\nu}^{2}-\omega_{f}^{2}} \overline{\mathrm{D}}_{\nu}=\frac{1}{\alpha_{\nu}} \frac{1}{\rho S} \frac{1}{\beta_{\nu}} I_{\nu}=C_{\nu}^{*} I_{\nu} .
$$

11. Substitute Eq. (5.16) in Eq. (5.8), hence

$$
u_{f}(x, t)=\sum_{\nu} u_{f ; \nu}(x, t)=\sum_{\nu} \bar{C}_{\nu} X_{\nu}(x) \exp \left(\mathrm{i} \omega_{f} t\right) .
$$

12. The final solution of the forced vibrations is given by

$$
u_{f}(x, t)=\sum_{j} u_{f ; j}(x, t) .
$$

As can be seen, the orthogonality condition of the modes is necessary to solve the forced vibration problem using the Fourier method.

\section{Conclusions}

In this paper, the orthogonality condition of the modes of the specific mechanical system is derived. The system consists of the simple supported beam, actuators and concentrated masses. Hence, the system may have the technical interpretation. All physical quantities of the system components are considered, among other things, the mass and stiffness of both actuators and glue layers. So, the condition may be directly applied to the solution of the beam active vibration reduction problem. This procedure is outlined in Sect. 5 .
Quite similar, the orthogonality condition of the modes for other boundary conditions and free combination of actuators and concentrated masses may be derived.

As pointed out in the paper, each active beam vibration reduction problem may be solved analytically, even if the dynamic effects of the actuators and glue are considered.

\section{References}

[1] L. Majkut, Latin Am. J. Solids Struct. 7, 423 (2010).

[2] H. Saito, K. Otomi, J. Sound Vibrat. 62, 257 (1979).

[3] M.J. Maurizi, P.M. Belles, J. Sound Vibrat. 150, 330 (1991).

[4] L. Ercoli, P.A.A. Laura, J. Sound Vibrat. 114, 519 (1987).

[5] W.H. Liu, F.H. Yeh, J. Sound Vibrat. 117, 555 (1987).

[6] P.M. Przybyłowicz, Monograph 197, Oficyna Wydawnicza PW, Warszawa 2002.

[7] J. Wiciak, Monograph 175, AGH, Kraków 2008 (in Polish)

[8] C.H. Hansen, S.D. Snyder, Active Control of Noise and Vibration, E\&FN SPON, London 1997.

[9] C.R. Fuller, S.J. Elliot, P.A. Nielsen, Active Control of Vibration, Academic Press, London 1997.

[10] M. Kozień, Monograph 331, PK, Kraków 2006 (in Polish).

[11] M.J. Croker, Handbook of Noise and Vibration Control, Wiley, New York 2007.

[12] F. Charette, A. Berry, C. Guigou, J. Intellig. Mater. Syst. Struct. 8, 513 (1998).

[13] J.A. Hernandes, S.F.M. Almeida, A. Nabarrete, Composite Struct. 49, 55 (2000).

[14] W.J. Sheu, R.T. Huang, C.C. Wang, Sensors Actuators $A$ 148, 115 (2008).

[15] M. Pietrzakowski, Monograph 204, Oficyna Wydawnicza PW, Warszawa 2004.

[16] A. Brański, Acoustic Waves, InTech, Rijeka, Croatia 2011, Ch. 18, p. 397.

[17] A. Brański, in: Proc. XVIII Conf. on Acoustic and Biomedical Engineering, Eds. Z. Damijan, J. Wiciak, Kraków-Zakopane (Poland) 2011, p. 102.

[18] S. Kasprzyk, M. Wiciak, Opuscula Mathematica 27, 245 (2007).

[19] C.N. Bapat, C. Bapat, J. Sound Vibrat. 112, 177 (1987).

[20] S. Kaliski, Vibrations and Waves, PWN, Warszawa 1986 (in Polish).

[21] C.W de Silva, Vibration, Fundamentals and Practice, CRC Press, Boca Raton 2000.

[22] K.H. Low, S. Naguleswaran, J. Sound Vibrat. 215, 381 (1998). 